\title{
Differentiation Between Combined Hepatocellular Carcinoma and Hepatocellular Carcinoma: Comparison of Diagnostic Performance Between Ultrasomics-Based Model and CEUS LIRADS v2017
}

\section{Chao-qun Li}

First Affiliated Hospital of Sun Yat-sen University

\section{Xin Zheng}

First Affiliated Hospital of Sun Yat-sen University

\section{Huan-ling Guo}

First Affiliated Hospital of Sun Yat-sen University

\section{Mei-qing Cheng}

First Affiliated Hospital of Sun Yat-sen University

\section{Yang Huang}

First Affiliated Hospital of Sun Yat-sen University

\section{Xiao-yan Xie}

First Affiliated Hospital of Sun Yat-sen University

\section{Ming-de Lu}

First Affiliated Hospital of Sun Yat-sen University

\section{Ming Kuang}

First Affiliated Hospital of Sun Yat-sen University

\section{Wei Wang}

First Affiliated Hospital of Sun Yat-sen University

\section{Li-da Chen ( $\nabla$ chenlda@mail.sysu.edu.cn )}

First Affiliated Hospital of Sun Yat-sen University

\section{Research Article}

Keywords: Combined hepatocellular cholangiocarcinoma, Hepatocellular carcinoma, Ultrasomics, Liver Imaging Reporting And Data System.

Posted Date: August 4th, 2021

DOI: https://doi.org/10.21203/rs.3.rs-654430/v1 
License: (c) (i) This work is licensed under a Creative Commons Attribution 4.0 International License. Read Full License

Version of Record: A version of this preprint was published at BMC Medical Imaging on March 3rd, 2022. See the published version at https://doi.org/10.1186/s12880-022-00765-x. 


\section{Abstract \\ Background.}

The imaging findings of combined hepatocellular cholangiocarcinoma $(\mathrm{CHC})$ may be similar to hepatocellular carcinoma (HCC). CEUS LI-RADS may not perform well in distinguishing $\mathrm{CHC}$ from HCC. Studies have shown that Radiomics has an excellent ability of imaging analysis. This study was to establish and confirm an ultrasomics model for differentiating $\mathrm{CHC}$ from HCC.

\section{Methods.}

Between 2004 and 2016, we retrospectively identified 53 eligible CHC patients and included 106 eligible $\mathrm{HCC}$ with a ratio of $\mathrm{HCC}: \mathrm{CHC}=2: 1$ randomly, all of which were categorized according to ContrastEnhanced (CE) ultrasonography (US) Liver Imaging Reporting and Data System (LI-RADS) version 2017. The model based on ultrasomics features of CE US was developed in $74 \mathrm{HCC}$ and $37 \mathrm{CHC}$, confirmed in $32 \mathrm{HCC}$ and $16 \mathrm{CHC}$. The diagnostic performance of LI-RADS or ultrasomics model was assessed by the area under the curve (AUC), accuracy, sensitivity and specificity.

\section{Results.}

In entire and validation cohort, $62.3 \%$ and $78.1 \%$ of HCC were correctly assigned to LR-5, $73.6 \%$ and $87.5 \%$ of $\mathrm{CHC}$ were assigned to LR-M correctly. Up to $27.4 \%$ of HCC and $26.4 \%$ of $\mathrm{CHC}$ were misclassified by CE US LI-RADS. $97.2 \%$ and $90.6 \%$ of $\mathrm{HCC}$ as well as $94.3 \%$ and $87.5 \%$ of $\mathrm{CHC}$ correctly diagnosed by the ultrasomics model in entire and validation cohort respectively. The AUC, accuracy, sensitivity, and specificity of ultrasomics model were significant improved compared with CE US LI-RADS in entire cohort ( all $P<0.05$ ), but there was no significant difference in validation cohort.

\section{Conclusion.}

The proposed ultrasomics model had an ability similar to CE US LI-RADS v2017 for differentiating CHC from $\mathrm{HCC}$, which may be more helpful to differentiate $\mathrm{CHC}$ from $\mathrm{HCC}$ in clinical diagnosis.

\section{Background}

Combined hepatocellular cholangiocarcinoma $(\mathrm{CHC})$ is an extremely rare primary liver cancer, which is composed of a mixture of hepatocellular carcinoma ( $\mathrm{HCC}$ ) and cholangiocarcinoma (CCA), with more aggressive behavior and worse prognosis than $\operatorname{HCC}$ or $\operatorname{CCA}[1,2,3,4]$. It is reported that the clinical characteristics of $\mathrm{CHC}$ patients are similar to those of $\mathrm{HCC}$, and $66 \%$ of $\mathrm{CHC}$ patients have common risk 
factors with $\mathrm{HCC}\left[{ }^{5}, 6\right]$. In addition, CT / MRI or Contrast-Enhanced (CE) ultrasonography (US), the imaging findings of $\mathrm{CHC}$ may be similar to either or both of $\mathrm{HCC}$ and $\mathrm{CCA}[3,7,8]$.

Serum markers alpha-fetoprotein (AFP) and carbohydrate antigen 19-9 (CA19-9) were not specific for $\mathrm{CHC}$, even the combination of imaging features and tumor markers as diagnostic criteria still indicated an inadequate diagnostic efficiency $[6,8,9]$. Studies proved that the incidence of lymph node metastasis in patients with $\mathrm{CHC}$ is higher than that in patients with $\mathrm{HCC}$, so curative surgery must be performed with systemic nodal dissection[6, ${ }^{10]}$, and localized treatments for HCC such as transarterial chemoembolization (TACE) were not an ideal treatment for $\mathrm{CHC}$ in theory $\left[{ }^{11}, 12\right]$. In addition, the role of liver transplantation currently remains uncertain in this disease $\left[{ }^{13}, 14\right]$. Imaging misdiagnosis of $\mathrm{CHC}$ as $\mathrm{HCC}$ could lead to nonstandard treatments for $\mathrm{CHC}$, the correct preoperative diagnosis is still essential.

Radiomics is an imaging analysis method based on high-throughput imaging features extracting from tomographic images [ ${ }^{15}$. In recent years, radiomics based on CT / MRI successfully showed favorable abilities in oncology research $[16,17,18]$. US was generally the preferred method for focal liver lesions (FLL) screening, and CE US had a high accuracy in the identification of same as CT / MRI [ ${ }^{19]}$. Study by Li Wei et al. indicated that ultrasound-based radiomics (ultrasomics) can improve the discrimination of significant liver fibrosis $\left[{ }^{20]}\right.$. Peng et al. had shown that the ultrasomics models was helpful to distinguish the histopathological subtypes of primary liver cancer[ ${ }^{21]}$. Hu et al. demonstrated that ultrasomics was potential biomarker for microvascular invasion prediction in $\mathrm{HCC}\left[{ }^{22}\right.$. In addition, it had been reported that ultrasomics had good performance in differentiating benign from malignant FLL, predicting tumor deposition and lymph node metastasis etc $[23,24,25,26]$.

There is no report on the application of ultrasomics methods in the identification of $\mathrm{CHC}$ and HCC. Our research aimed to develop and validate a ultrasomics model to distinguish between $\mathrm{HCC}$ and $\mathrm{CHC}$, and diagnostic performance of CEUS LI-RADS version 2017 was compared with those of ultrasomics model.

\section{Methods}

\section{Patients}

The study was approved by the ethical committee of our institution, and informed consent was obtained. Our retrospective study was conducted all eligible $\mathrm{CHC}$ patients on the basis of following inclusion criteria between 2004 and 2016. HCC patients, who met following inclusion criteria during this period, were randomly included in our study with a ratio of $\mathrm{HCC}: \mathrm{CHC}=2: 1$. The inclusion criteria were as follows: (1) primary $\mathrm{HCC}$ or $\mathrm{CHC}$ diagnosed histopathologically after biopsy or surgery, (2) patients with high risk for HCC (cirrhosis or chronic hepatitis viral infection), (3) Available CEUS examination performed 2 weeks before operation.

The exclusion criteria were as follows: (1) unavailability of histological evaluation by surgery or biopsy, (2) incomplete clinic-pathological data or CE US data. 
The flow chart of the study population was presented in Figure 1.

\section{US imaging acquisition}

US studies were performed first for scanning entire liver by experienced radiologist with the following equipment: (1) Aplio SSA-770 or Aplio 500 (Toshiba Medical Systems, Tokyo, Japan) with a 375BT convex transducer with frequency range, of 1.9 to $6.0 \mathrm{MHz}$. (2) Acuson Sequoia 512 (Siemens Medical Solutions, Mountain View, CA, United States) with a 4V1 vector transducer with frequency range of 1.0 to 4.0 MHz. (3) Aixplorer Ultrasound system (SuperSonic Imagine, Aix-en-Provence, France) equipped with the SC6-1 convex probe with frequency range of 1.0 to $6.0 \mathrm{MHz}$. If patients had multiple liver lesions, the largest one was regarded as target lesion. After identifying the target lesion and storing images recorded size, location, echo, shape, boundary, and margin, CE US examination with the same probe was performed after administration of $1.2-2.4 \mathrm{~mL}$ of SonoVue (Bracco Imaging, Milan, Italy) within 1-2 seconds into the antecubital vein followed by a $5 \mathrm{~mL}$ normal saline flush. The target lesion were observed continuously for at least 5 minutes for recording CE US features. Arterial phase hyperenhancement is described as entirely or partially (not rimlike and peripheral discontinuous globular) hyperechoic compared with the surrounding parenchyma. Washout is described as occurring of hypoechoic relative to liver after hyperechoic or isoechoic during the arterial phase. Early washout is defined as that occurs within 60s after injection of the contrast agent, and marked washout is defined when punched-out appearance (markedly hypoechoic emerging black) appears within 2 minutes.

\section{CE US LI-RADS categories}

The records of the whole process of CE US examination were independently analyzed by two experienced radiologists (reader 1 and reader 2, not involved in US examinations) with more than 8 years of experience in CE US. The discussion will focus on the cases where two readers have different opinions until the final consensus is reached. All of them were blind to the pathological and other imaging information. They were asked to classify into CE US LR-1 to LR-5 or LR-M according to CE US LI-RADS v2017. CE US LR-5 (not rim and peripheral discontinuous globular APHE with late and mild washout, meanwhile nodules size $\geq 10 \mathrm{~mm}$ ) is defined as HCC. Then the evaluation of diagnostic performance of v2017 LI-RADS would be conducted for HCC and CHC.

\section{Ultrasomics features extraction and ultrasomics models}

Images of each lesion confirmed by two radiologists in consensus (including 4 images from baseline US, arterial, portal venous and late phases of CE US, respectively) were used to delineate a region of interest (ROI) around the outline of the tumor using ITK-SNAP software (version 3.6.0; www.itksnap.org). ROI of each image included $1 \mathrm{~cm}$ around the lesion margin, but not the portion beyond the liver parenchyma. Then 5936 features were extracted from one single ROI (A total of 23744 features from each patient) using the Ultrasomics-Platform (Version 2.1, Ultrasomics Artificial Intelligence X-lab, Guangzhou), which mainly contains two major functions of ultrasomics feature mining and machine learning for model building. It is a kind of software for medical research including essential four modules of segmentation, 
calculation, feature selection and machine learning, and based on automatic analysis of the heterogeneity of the ROI, the clinical prediction is finally achieved through the above key processes. Finally, A ultrasomics model was developed based on features selected by spearman rank correlation analysis and machine-learning algorithms of support vector machine using software, and a ultrasomics score (U-score) was calculated by the ultrasomics model (U model) for each patient. The optimal cut-off value for $\mathrm{U}$ model were determined using receiver operating curve (ROC) analysis. HCC was defined as a U-score of each lesion greater than optimal cut-off value.

$\mathrm{CHC}$ and $\mathrm{HCC}$ patients finally included in this study were respectively grouped into training cohort and validation cohort at a ratio of $7: 3$ randomly. $U$ model was developed in training cohort, confirmed in validation cohort.

\section{Statistical analysis}

Continuous variables were expressed as means \pm standard deviations. Categorical variables were reported as numbers and percentage, compared by the chi-square test. The optimal cut-off values for $U$ model were determined using ROC analysis. The diagnostic performance of LI-RADS or U model was assessed by ROC and the area under the curve (AUC), accuracy, sensitivity, specificity with $95 \%$ confident interval $(\mathrm{Cl})$. Delong's test was used to compare the statistical differences between any two AUCs.

Statistical analysis was performed with SPSS 22.0 for Windows (Chicago, IL) and Ultrasomics-Platform (Version 2.1, Ultrasomics Artificial Intelligence X-lab, Guangzhou). $P<0.05$ was considered statistically significant.

\section{Results}

\section{Characters of patients and lesions}

The final entire study cohort consisted of 159 patients $(\mathrm{HCC}=106 ; \mathrm{CHC}=53)$, separated into training cohort $(\mathrm{n}=111, \mathrm{HCC}=74, \mathrm{CHC}=37)$ and validation cohort $(\mathrm{n}=48, \mathrm{HCC}=32, \mathrm{CHC}=16)$ randomly. The basic characteristics of all patients and lesions are shown in Table 1. There was no significant difference in clinical and pathological characteristics between $\mathrm{HCC}$ and $\mathrm{CHC}(P>0.05)$, except the levels of CA125 and CEA in $\mathrm{CHC}$ was significantly higher than that in $\mathrm{HCC}(P<0.05)($ Table 1$)$.

\section{LI-RADS categories and diagnostic performance}

In entire cohort, 66, 2, 29 and 9 of $106 \mathrm{HCC}$ lesions were assigned to LR-5, LR-4, LR-M and LR-TIV respectively, while 25, 0,5 and 2 of $32 \mathrm{HCC}$ in validation cohort were same assigned (Table 1). $62.3 \%$ and $78.1 \%$ of HCC in entire and validation cohort were assigned to LR-5, and most of the rest of HCC (27.4\% and $15.6 \%$ ) were assigned to LR-M. $73.6 \%$ and $87.5 \%$ of $\mathrm{CHC}$ in entire and validation cohort were assigned to LR-M, and all of the rest of CHC were assigned to LR-5 (Table 1). No HCC was assigned to LR-1هLR-3 and no CHC was assigned to LR-1 1 LR-4 and LR-TIV. The accuracy, sensitivity, specificity of CE US LI-RADS were $66.0 \%$ (95\% Cl: 58.7 to 73.4 ), 62.3\% (95\% Cl: 52.3 to 71.5$), 73.6 \%$ (95\% Cl: 59.7 to 84.7 ) 
and $81.3 \%$ (95\% Cl: 70.2 to 92.3 ), $78.1 \%$ (95\% Cl: 60.0 to 90.7), 81.3\% (95\% Cl: 54.4 to 96.0) in entire cohort and validation cohort, respectively (Table 2). The AUC of the CE US LI-RADS (LR-5 as a predictor of $\mathrm{HCC}$ ) was 0.679 (95\% Cl: 0.601 to 0.751$)$ and 0.797 (95\% Cl: 0.656 to 0.899$)$ in entire cohort and validation cohorts respectively (Table 2).

\section{Diagnostic performance of ultrasomics model}

The U-score of HCC ranged from 0.004093 to 1.531201 and from 0.004093 to 1.513874 in entire and validation cohorts respectively, while the U-score of non-HCC ranged from -1.17112 to -0.0395 and from -0.99242 to -0.0395 . The optimal cut-off value for $U$ model obtained by using ROC analysis was -0.0395 . $\mathrm{HCC}$ was defined as a U-score $>-0.0395$ by U model, otherwise $\mathrm{CHC}$ was defined.

There were 103 of 106 (97.2\%) HCC and 50 of 53 (94.3\%) CHC correctly diagnosed by the U model, 29 of $32(90.6 \%) \mathrm{HCC}$ and 14 of 16 (87.5\%) CHC in validation cohort correctly diagnosed as well. Using U model, an increased cases of $34.9 \%$ of HCC (37 of 106) in LR-4 / M / TIV in entire cohort was accurately diagnosed than CE US LI-RADS v2017, meanwhile all of CHC misclassified to LR-5 was accurately diagnosed (Table 3). Only 3 HCC (2 assigned to LR-5, 1 assigned to LR-M) and 3 CHC (all assigned to LRM) were not confirmed by the $U$ model in entire cohort, and 3 HCC (2 assigned to LR-5, 1 assigned to LR$\mathrm{M}$ ) as well as $2 \mathrm{CHC}$ (all assigned to LR-M) in validation cohort (Table 3). CE US images of two cases correctly diagnosed by U model and wrongly diagnosed by CE US LI-RADS v2017 were presented in Figure 2 and Figure 3.

The accuracy of the $\mathrm{U}$ model increased to $96.2 \%$ (95\% Cl: 93.3 to 99.2$)$ and $90.0 \%$ (95\% Cl: 80.9 to 98.2 ) in entire cohort and validation cohorts respectively $(P<0.001 ; P=0.386)$. The AUC $(0.981[95 \% \mathrm{Cl}, 0.946$ to 0.996$])$ of the U model in entire cohort was higher than CE US LI-RADS v2017 $(P<0.001)$ (Table 2). There was no significant difference in AUC between ultrasomics and CE US LI-RADS v2017 in validation cohort $(P=0.300)$. The sensitivity, and specificity of the U model were $97.2 \%$ (95\% Cl: 92.0 to 99.4$), 96.2 \%$ (95\% Cl: 87.0 to 99.5$)$ and $90.6 \%$ (95\% Cl: 75.0 to 98.0$), 87.5 \%$ (95\% Cl: 61.7 to 98.4 ) in entire cohort and validation cohort, respectively. The sensitivity and specificity in entire cohort were significantly improved by using the $U$ model (all $P<0.05$ ) (Table 2). Although the sensitivity and specificity by using the U model in validation cohort were higher than CE US LI-RADS v2017, the difference were statistically insignificant (all $P>0.05$ ) (Table 2).

\section{Discussion}

In our study, we developed and validated an ultrasomics model for distinguishing between HCC and CHC, which proved promising differentiation ability and reliability similar to CE US LI-RADS v2017.

Previous studies on $\mathrm{CHC}$ mostly were keen on clinical and pathological characteristics or description of imaging features, but the existing tumor biomarkers and imaging techniques are still insufficient to correctly differentiate $\mathrm{CHC}$ from $\mathrm{HCC}[8,9, \ldots$, . Tian et al. constructed a risk prediction model of $\mathrm{CHC}$ based on demographic, clinical and imaging characteristics, which presented good discrimination, but its 
intention was not to be a diagnostic test ${ }^{\square}$. Two-thirds of $\mathrm{CHC}$ patients by enhanced $\mathrm{CT} / \mathrm{MR}$ underwent imaging misdiagnosis, and the sensitivity was as low as $34 \%$, . Further researches proved the moderate sensitivity and specificity (61-71\% and 75-85\%) of using CT/MR LI-RADS to correctly categorize CHC as LR-M, and misclassification as HCC in approximately $50 \%$ of $\mathrm{CHC}_{\text {"] }}^{[}$. Radiomics based on CT / MRI have showed favorable performance in differentiation of tumors, prediction for tumor microvascular invasion, lymph node metastasis, early recurrence and prognosis[16,17,18]. Zhang et al. established and validated a radiomics-based model with favorable ability for ICC differentiation of $\mathrm{CHC}^{\square}$. Study by Liu et al. proved promising performance of MRI radiomics features in distinguishing $\mathrm{CHC}$ from $\mathrm{HCC}$ and CCA, but CT was of limited value and the study included a small sample of only $24 \mathrm{CHC}, 24 \mathrm{CCA}$ and $38 \mathrm{HCC}$, which need more future validation ${ }^{\square}$. There still existed no definite standard to distinguish patients with $\mathrm{CHC}$ from $\mathrm{HCC}$, and there are few reports on the identification of $\mathrm{CHC}$ and $\mathrm{HCC}$ by CE US.

Based on our data, we found there was significant difference only in CA125 and CEA of all clinicopathologic characteristics between $\mathrm{HCC}$ and $\mathrm{CHC}(P<0.05)$. While there was no significant difference in AFP, that means these tumor markers are not specific for definitive diagnosis of $\mathrm{CHC}$. Our data shown that HCC was most likely to be misclassified to LR-M (up to $27.4 \%$ ), and $\mathrm{CHC}$ was most likely to be misclassified to LR-5 (up to $26.4 \%$ ) by CEUS LI-RADS v2017. The moderate sensitivity and specificity of using LI-RADS (62.3\%, 73.6\% and 78.1\%, 81.3\% in entire and validation cohort respectively) in correctly classifying CHC as LR-M in our study were similar to previous studies[35]. These means that it is difficult to distinguish HCC from CHC by CEUS LI-RADS. So we tried to use ultrasomics features for $\mathrm{CHC}$ differentiation of HCC, and comparative assessment of diagnostic performance between ultrasomics and CE US LI-RADS v2017 would be conduct.

In our study, an increased case of $34.9 \%$ of HCC (37 of 106) and $20.8 \%$ of $\mathrm{CHC}$ (11 of 53) in entire cohort was accurately diagnosed by using $\mathrm{U}$ model, and HCC accurately diagnosed by using $\mathrm{U}$ model increased $12.5 \%$ (4 of 32) while same CHC accurately diagnosed as LI-RADS in validation cohort. $26.4 \% \mathrm{CHC}$ (14 of 53) were misdiagnosed as HCC and $27.4 \%$ HCC (29 of 106) were misdiagnosed as non-HCC malignancy in entire cohort by CE US LI-RADS, which means that CE US LI-RADS is inadequate to differentiate CHC from HCC. All HCC in LR-4 / TIV as well as $96.5 \%$ HCC in LR-M and all CHC in LR-5 accurately diagnosed by using $U$ model. The accuracy of the $U$ model improved significantly in entire cohort $(P<0.01)$. The ultrasomics features had an ability similar to CE US LI-RADS v2017 for differentiating $\mathrm{CHC}$ from HCC due to higher or similar AUC, sensitivity and specificity of $U$ model in the entire cohort (all $P<0.05)$ and validation cohort (all $P>0.05$ ). Although our results, like those of the study about radiomics in differentiating $\mathrm{CHC}$ from $\mathrm{CHC}$ by Liu et al, were not perfect, high AUC, sensitivity and specificity of the $U$ model indicated promising discrimination ability and accuracy. We speculated the reason for no significant difference in diagnostic performance between ultrasomics and CE US LI-RADS v2017 in validation cohort might be the small sample in our study. Because the AUC of the training cohort used to build the $U$ model was 1 , we compare the performance of $U$ model bettween the entire cohort and the validation cohort. In general, performance of this model in distinguishing $\mathrm{CHC}$ from $\mathrm{HCC}$ is superior to that in LI-RADS v2017, it may be more helpful in clinical practice for CHC inpatients. 
There are limitations in our study. First, the sample size of $\mathrm{CHC}$ participants in our study is not large. Second, our data from a single-center, the results need to be expanded to other centers and confirmed its reproducibility. Third, US is operator-dependent, and also has lower sensitivity in overweight and obese patients and all the potential nodules may not be found with cross-sectional imaging by US. Finally, there is the possibility of overfitting during the model development.

\section{Conclusions}

In conclusion, we developed a ultrasomics model for preoperative differentiation of $\mathrm{CHC}$ from $\mathrm{HCC}$, which had an ability similar to CE US LI-RADS v2017 for differentiating CHC from HCC. This model may be more helpful to differentiate $\mathrm{CHC}$ from HCC in clinical diagnosis.

\section{Abbreviations}

$\mathrm{CHC}$

combined hepatocellular cholangiocarcinoma, $\mathrm{HCC}=$ hepatocellular carcinoma, $\mathrm{CCA}=$ cholangiocarcinoma, CE = contrast enhanced, LI-RADS = Liver Imaging Reporting and Data System, CA19$9=$ carbohydrate antigen $19-9, \mathrm{AFP}=$ Alpha-fetoprotein, TACE $=$ transarterial chemoembolization, $\mathrm{FLL}=$ focal liver lesions, $\mathrm{ROI}=$ region of interest, $\mathrm{U}$-score $=$ ultrasomics score, $\mathrm{U}$ model $=$ ultrasomics model, $\mathrm{ROC}$ $=$ receiver operating curve, $\mathrm{AUC}=$ the area under the curve, $\mathrm{Cl}=$ confidence interval, $\mathrm{TIV}=$ tumor in vein, PPV = positive predictive value, NPV = negative predictive value.

\section{Declarations}

\section{Ethics approval and consent to participate:}

This study has been approved by the Institutional Review Board of the First Affiliated Hospital of Sun Yatsen University, and informed consent was obtained. All methods in this study were carried out in accordance with relevant guidelines and regulations.

\section{Consent for publication:}

All data including ultrasound imaging in this study have been approved for publication.

\section{Availability of data and materials:}

The datasets used and analysed during the current study are available from the corresponding author on reasonable request.

\section{Competing interest:}

The authors do not have any conflict of interest to declare. 


\section{Funding:}

This work was supported by the National Nature Science Foundation of China (No: 81971630), and the Guangzhou Science and Technology Foundation (No: 201904010187).

The funder (Wei Wang) participated in the study concepts and manuscript review and editing, and supported the work of data collection.

\section{Acknowledgements:}

None.

\section{Authors' contributions:}

Chao-qun Li (study concepts and design, data acquisition, literature research, data analysis, manuscript draft and editing); Xin Zheng ( study concepts and design, data acquisition, data analysis, clinical studies, manuscript draft and editing); Huan-ling Guo (study concepts and design, clinical studies, data analysis, manuscript review and editing); Mei-qing Cheng (study concepts and design, data analysis, manuscript review and editing); Yang Huang (study concepts and design, methodology, literature research, manuscript review and editing); Xiao-yan Xie (study concepts and design, data resources, manuscript review and editing); Ming-de Lu and Ming Kuang (study concepts and design, methodology, manuscript review and editing); Wei Wang (study concepts and design, acquisition of funding, manuscript review and editing); Li-da Chen (study concepts and design, clinical studies, data analysis, manuscript draft and editing, supervision of the research group ).

all authors have read and approved the manuscript.

\section{References}

1. He C, Mao Y, Wang J, Song Y, Huang X, Lin X, et al. The Predictive Value of Staging Systems and Inflammation Scores for Patients with Combined Hepatocellular Cholangiocarcinoma After Surgical Resection: a Retrospective Study. J Gastrointest Surg. 2018;22(7):1239-1250.

2. Kim SH, Park YN, Lim JH, Choi GH, Choi JS, Kim KS. Characteristics of combined hepatocelluarcholangiocarcinoma and comparison with intrahepatic cholangiocarcinoma. Eur J Surg Oncol. 2014;40(8):976-81.

3. Maximin S, Ganeshan DM, Shanbhogue AK, Dighe MK, Yeh MM, Kolokythas O, et al. Current update on combined hepatocellular-cholangiocarcinoma. Eur J Radiol Open. 2014;1:40-48.

4. Zuo HQ, Yan LN, Zeng Y, Yang JY, Luo HZ, Liu JW, et al. Clinicopathological characteristics of 15 patients with combined hepatocellular carcinoma and cholangiocarcinoma. Hepatobiliary Pancreat Dis Int. 2007;6(2):161-5. 
5. Cazals-Hatem D, Rebouissou S, Bioulac-Sage P, Bluteau O, Blanché H, Franco D, et al. Clinical and molecular analysis of combined hepatocellular-cholangiocarcinomas. J Hepatol. 2004;41(2):292298.

6. Kassahun WT, Hauss J. Management of combined hepatocellular and cholangiocarcinoma. Int J Clin Pract. 2008;62(8):1271-1278.

7. Lipsett PA, Pitt HA, Colombani PM, Boitnott JK, Cameron JL. Choledochal cyst disease. A changing pattern of presentation. Ann Surg. 1994;220(5):644-652.

8. Huang XW, Huang Y, Chen LD, Wang Z, Yang Z, Liu JY, et al. Potential diagnostic performance of contrast-enhanced ultrasound and tumor markers in differentiating combined hepatocellularcholangiocarcinoma from hepatocellular carcinoma and cholangiocarcinoma. J Med Ultrason. 2018;45(2):231-241.

9. Panjala C, Senecal DL, Bridges MD, Kim GP, Nakhleh RE, Nguyen JH, et al. The diagnostic conundrum and liver transplantation outcome for combined hepatocellular-cholangiocarcinoma. Am J Transplant. 2010;10(5):1263-1267.

10. Liver Cancer Study Group of Japan. Primary liver cancer in Japan. Clinicopathologic features and results of surgical treatment. Ann Surg. 1990;211(3):277-287.

11. Kim KH, Lee SG, Park EH, Hwang S, Ahn CS, Moon DB, et al. Surgical treatments and prognoses of patients with combined hepatocellular carcinoma and cholangiocarcinoma. Ann Surg Oncol. 2009;16(3):623-629.

12. Wang J, Wang F, Kessinger A. Outcome of combined hepatocellular and cholangiocarcinoma of the liver. J Oncol. 2010;2010:917356.

13. Panjala C, Senecal DL, Bridges MD, Kim GP, Nakhleh RE, Nguyen JH, et al. The diagnostic conundrum and liver transplantation outcome for combined hepatocellular-cholangiocarcinoma. Am J Transplant. 2010;10(5):1263-1267.

14. Perkins JD. Transplanted patients with combined hepatocellular-cholangiocarcinoma: now what?. Liver Transpl. 2007;13(10):1465-1466.

15. Gillies RJ, Kinahan PE, Hricak H. Radiomics: Images Are More than Pictures, They Are Data. Radiology. 2016;278(2):563-577.

16. Banerjee S, Wang DS, Kim HJ, Sirlin CB, Chan MG, Korn RL, et al. A computed tomography radiogenomic biomarker predicts microvascular invasion and clinical outcomes in hepatocellular carcinoma. Hepatology. 2015;62(3):792-800.

17. Huang YQ, Liang CH, He L, Tian J, Liang CS, Chen X, et al. Development and Validation of a Radiomics Nomogram for Preoperative Prediction of Lymph Node Metastasis in Colorectal Cancer. J Clin Oncol. 2016;34(18):2157-2164.

18. Shan QY, Hu HT, Feng ST, Peng ZP, Chen SL, Zhou Q, et al. CT-based peritumoral radiomics signatures to predict early recurrence in hepatocellular carcinoma after curative tumor resection or ablation. Cancer imaging. 2019;19(1):11. 
19. Kim TK, Noh SY, Wilson SR, Kono Y, Piscaglia F, Jang HJ, et al. Contrast-enhanced ultrasound (CEUS) liver imaging reporting and data system (LI-RADS) 2017 - a review of important differences compared to the CT/MRI system. Clin Mol Hepatol. 2017;23(4):280-289.

20. Li W, Huang Y, Zhuang BW, Liu GJ, Hu HT, Li X, et al. Multiparametric ultrasomics of significant liver fibrosis: A machine learning-based analysis. Eur Radiol. 2019;29(3):1496-1506.

21. Peng Y, Lin P, Wu L, Wan D, Zhao Y, Liang L, et al. Ultrasound-Based Radiomics Analysis for Preoperatively Predicting Different Histopathological Subtypes of Primary Liver Cancer. Front Oncol. 2020;10:1646.

22. Hu HT, Wang Z, Huang XW, Chen SL, Zheng X, Ruan SM, et al. Ultrasound-based radiomics score: a potential biomarker for the prediction of microvascular invasion in hepatocellular carcinoma. Eur Radiol. 2019;29(6):2890-2901.

23. Chen LD, Li W, Xian MF, Zheng X, Lin Y, Liu BX, et al. Preoperative prediction of tumour deposits in rectal cancer by an artificial neural network-based US radiomics model. Eur Radiol. 2020;30(4):19691979.

24. Wang W, Zhang JC, Tian WS, Chen LD, Zheng Q, Hu HT, et al. Shear wave elastography-based ultrasomics: differentiating malignant from benign focal liver lesions. Abdom Radiol (NY). 2021;46(1):237-248.

25. Tong Y, Li J, Huang Y, Zhou J, Liu T, Guo Y, et al. Ultrasound-Based Radiomic Nomogram for Predicting Lateral Cervical Lymph Node Metastasis in Papillary Thyroid Carcinoma. Acad Radiol. 2020;S1076-6332(20)30440-2.

26. Yu FH, Wang JX, Ye XH, Deng J, Hang J, Yang B. Ultrasound-based radiomics nomogram: A potential biomarker to predict axillary lymph node metastasis in early-stage invasive breast cancer. Eur $\mathrm{J}$ Radiol. 2019;119:108658.

27. Jarnagin WR, Weber S, Tickoo SK, Koea JB, Obiekwe S, Fong Y, et al. Combined hepatocellular and cholangiocarcinoma: demographic, clinical, and prognostic factors. Cancer. 2002;94(7):2040-2046.

28. Yin X, Zhang BH, Qiu SJ, Ren ZG, Zhou J, Chen XH, et al. Combined hepatocellular carcinoma and cholangiocarcinoma: clinical features, treatment modalities, and prognosis. Ann Surg Oncol. 2012;19(9):2869-2876.

29. Koh KC, Lee H, Choi MS, Lee JH, Paik SW, Yoo BC, et al. Clinicopathologic features and prognosis of combined hepatocellular cholangiocarcinoma. Am J Surg. 2005;189(1):120-125.

30. Choi SH, Lee SS, Park SH, Kim KM, Yu E, Park Y, et al. LI-RADS Classification and Prognosis of Primary Liver Cancers at Gadoxetic Acid-enhanced MRI. Radiology. 2019;290(2):388-397.

31. Tian MX, He WJ, Liu WR, Yin JC, Jin L, Tang Z, et al. A Novel Risk prediction Model for Patients with Combined Hepatocellular-Cholangiocarcinoma. J Cancer. 2018;9(6):1025-1032.

32. Nishie A, Yoshimitsu K, Asayama Y, Irie H, Aibe H, Tajima T, et al. Detection of combined hepatocellular and cholangiocarcinomas on enhanced CT: comparison with histologic findings. AJR Am J Roentgenol. 2005;184(4):1157-1162. 
33. Fowler KJ, Sheybani A, Parker RA 3rd, Doherty S, M Brunt E, Chapman WC, et al. Combined hepatocellular and cholangiocarcinoma (biphenotypic) tumors: imaging features and diagnostic accuracy of contrast-enhanced CT and MRI. AJR Am J Roentgenol. 2013;201(2):332-339.

34. Jeon SK, Joo I, Lee DH, Lee SM, Kang HJ, Lee KB, et al. Combined hepatocellular cholangiocarcinoma: LI-RADS v2017 categorisation for differential diagnosis and prognostication on gadoxetic acid-enhanced MR imaging. Eur Radiol. 2019;29(1):373-382.

35. Ludwig DR, Fraum TJ, Cannella R, Ballard DH, Tsai R, Naeem M, et al. Hepatocellular carcinoma (HCC) versus non-HCC: accuracy and reliability of Liver Imaging Reporting and Data System v2018. Abdom Radiol (NY). 2019;44(6):2116-2132.

36. Potretzke TA, Tan BR, Doyle MB, Brunt EM, Heiken JP, Fowler KJ. Imaging Features of Biphenotypic Primary Liver Carcinoma (Hepatocholangiocarcinoma) and the Potential to Mimic Hepatocellular Carcinoma: LI-RADS Analysis of CT and MRI Features in 61 Cases. AJR Am J Roentgenol. 2016;207(1):25-31.

37. Zhang J, Huang Z, Cao L, Zhang Z, Wei Y, Zhang X, et al. Differentiation combined hepatocellular and cholangiocarcinoma from intrahepatic cholangiocarcinoma based on radiomics machine learning. Ann Transl Med. 2020;8(4):119.

38. Liu X, Khalvati F, Namdar K, Fischer S, Lewis S, Taouli B, et al. Can machine learning radiomics provide pre-operative differentiation of combined hepatocellular cholangiocarcinoma from hepatocellular carcinoma and cholangiocarcinoma to inform optimal treatment planning?. Eur Radiol. 2021;31(1):244-255.

\section{Tables}


Table 1

Basic characteristics of all patients and lesions with CE US LI-RADS classification

\begin{tabular}{|c|c|c|c|}
\hline Characteristics & $\mathrm{HCC}(n=106)$ & $\mathrm{CHC}(n=53)$ & $P$ value \\
\hline Gender & & & 0.778 \\
\hline Male & $89(84.0)$ & $42(79.2)$ & \\
\hline Female & $17(16.0)$ & $11(20.8)$ & \\
\hline Age (years)* & $55.3 \pm 10.9(29-76)$ & $53.8 \pm 10.6(25-80)$ & 0.384 \\
\hline Size & & & 0.201 \\
\hline$<3 \mathrm{~cm}$ & $15(14.2)$ & $3(5.7)$ & \\
\hline $3-5 \mathrm{~cm}$ & $38(35.8)$ & $15(28.3)$ & \\
\hline$>5 \mathrm{~cm}$ & $53(50.0)$ & $35(66.0)$ & \\
\hline Multiple lesions & 13 (12.3) & $13(24.5)$ & 0.068 \\
\hline AFP > $20(\mu \mathrm{g} / \mathrm{L})$ & $70(66.0)$ & $30(56.6)$ & 0.324 \\
\hline CA199 > 35 (U/mL) & $37(34.9)$ & $10(18.9)$ & 0.057 \\
\hline CA125 > 35 (U/mL) & $8(7.5)$ & $16(30.1)$ & $<0.001$ \\
\hline $\mathrm{CEA}>5(\mathrm{U} / \mathrm{mL})$ & $7(6.6)$ & $13(24.5)$ & 0.003 \\
\hline \multicolumn{4}{|c|}{ LI-RADS classification } \\
\hline LR-4 & 2 & 0 & \\
\hline LR-5 & 66 & 14 & \\
\hline LR-M & 29 & 39 & \\
\hline LR-TIV & 9 & 0 & \\
\hline \multicolumn{4}{|c|}{ Note.-- Unless otherwise indicated, data are number of cases, with percentages in parentheses. } \\
\hline * Data are means $\pm s$ & eviations, with range & rentheses & \\
\hline
\end{tabular}


Table 2

Diagnostic Performance of CE US LI-RADS and Ultrasomics

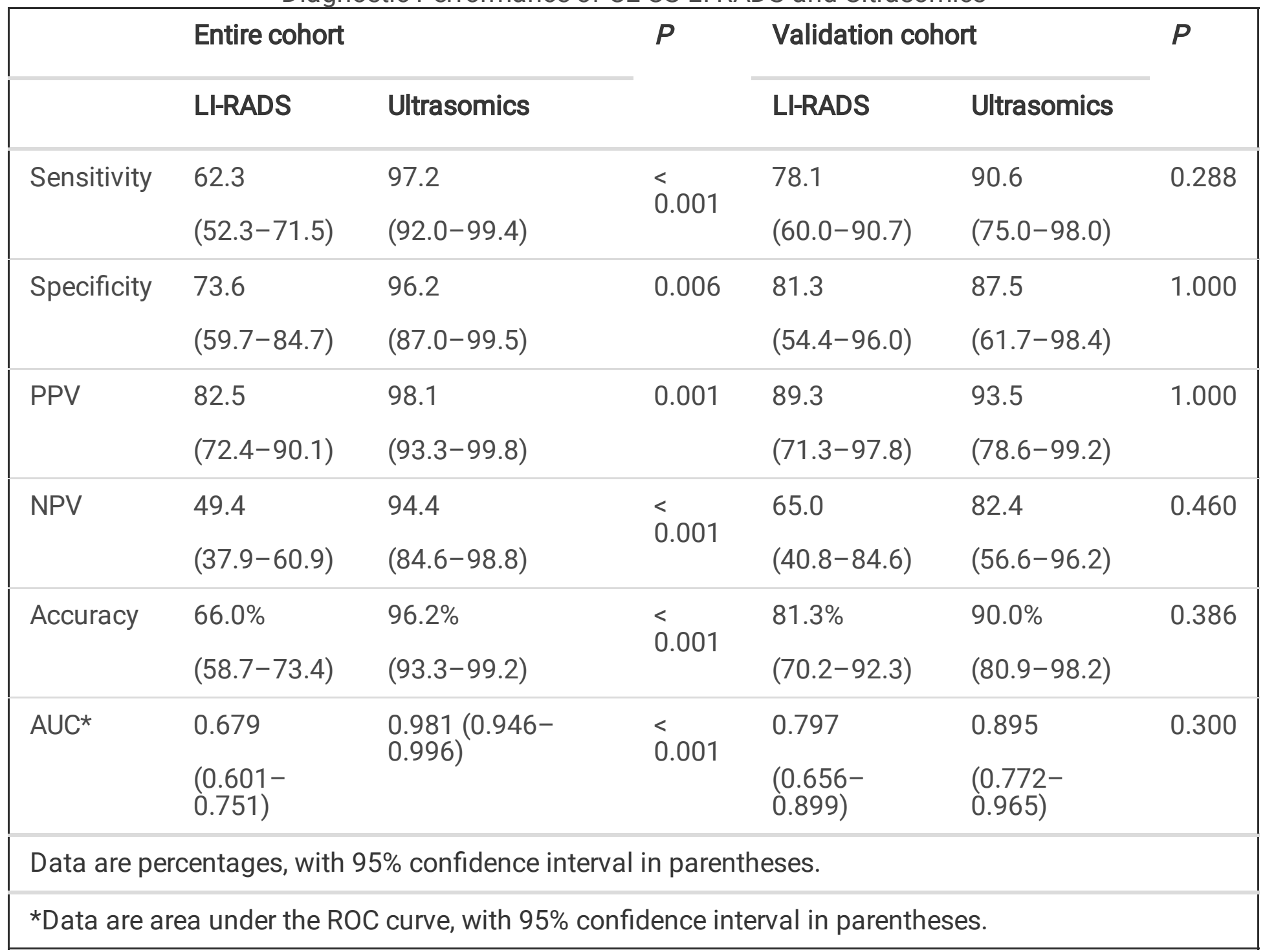


Table 3

CE US LI-RADS classification in cases with diagnosis of ultrasomics and pathology

\begin{tabular}{|lllllll|}
\hline & Pathology & Ultrasomics & LR-4 & LR-5 & LR-M & LR-TIV \\
\hline Entire cohort & HCC & HCC & 2 & 64 & 28 & 9 \\
\cline { 3 - 7 }$(159)$ & & Non-HCC & 0 & 2 & 1 & 0 \\
& CHC & HCC & 0 & 0 & 3 & 0 \\
\cline { 3 - 7 } & & Non-HCC & 0 & 14 & 36 & 0 \\
Validation cohort & HCC & HCC & 0 & 23 & 4 & 2 \\
$(48)$ & & Non-HCC & 0 & 2 & 1 & 0 \\
& & HCC & 0 & 0 & 2 & 0 \\
& CHC & Hon-HCC & 0 & 2 & 12 & 0 \\
\hline
\end{tabular}

Figures

Excluded

1. Without CE US examination $(\mathrm{n}=11)$

2. Incomplete clinic-pathological data $(n=6)$

Collecting data of eligible patients with histopathologically confirmed CHC from 2004 $2016(n=70)$

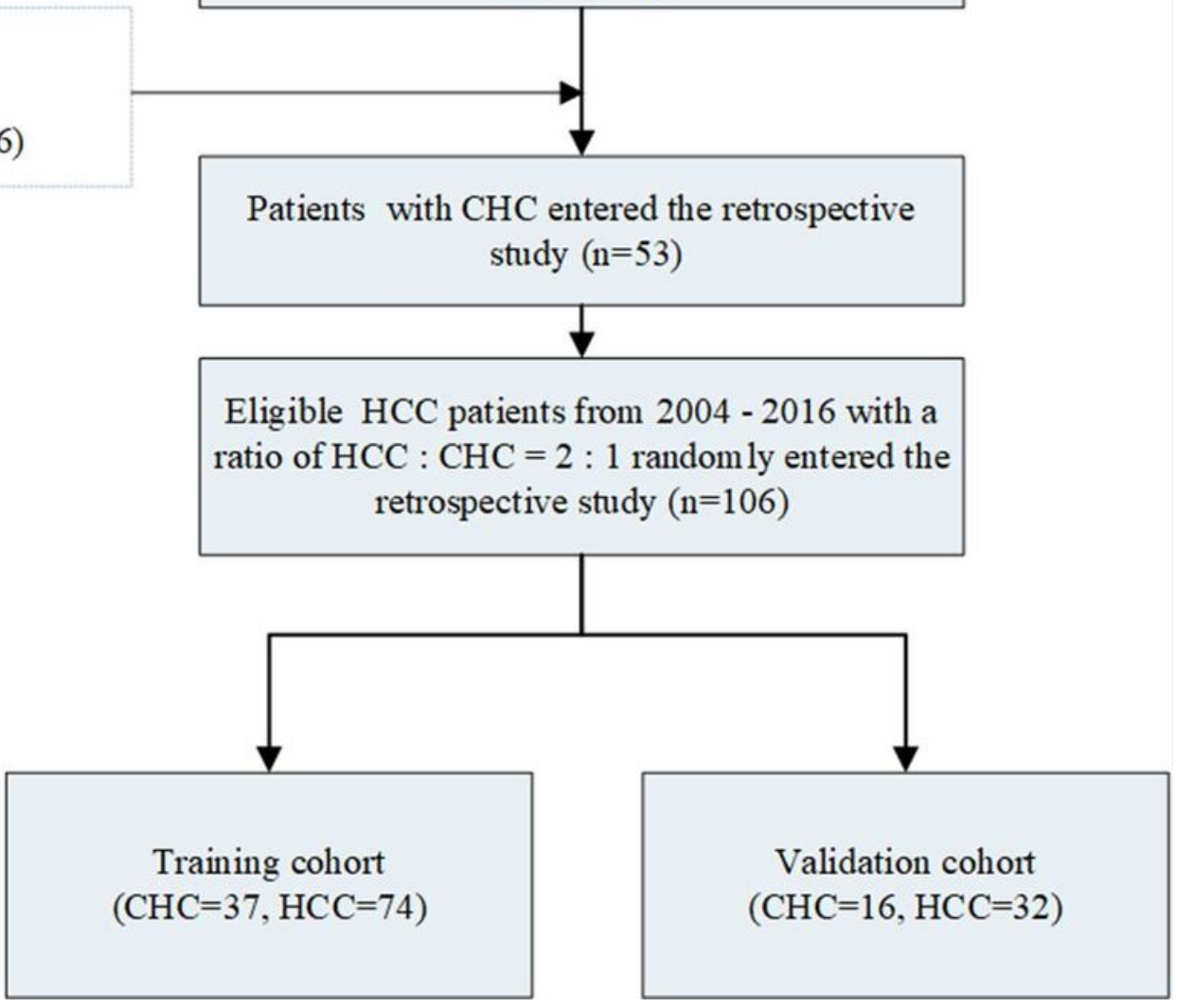

Figure 1 
Flowchart of $\mathrm{CHC}$ and HCC patients' enrollment. $\mathrm{CHC}=$ combined hepatocellular cholangiocarcinoma, HCC = hepatocellular carcinoma, CE = contrast enhanced, LI-RADS = Liver Imaging Reporting and Data System.

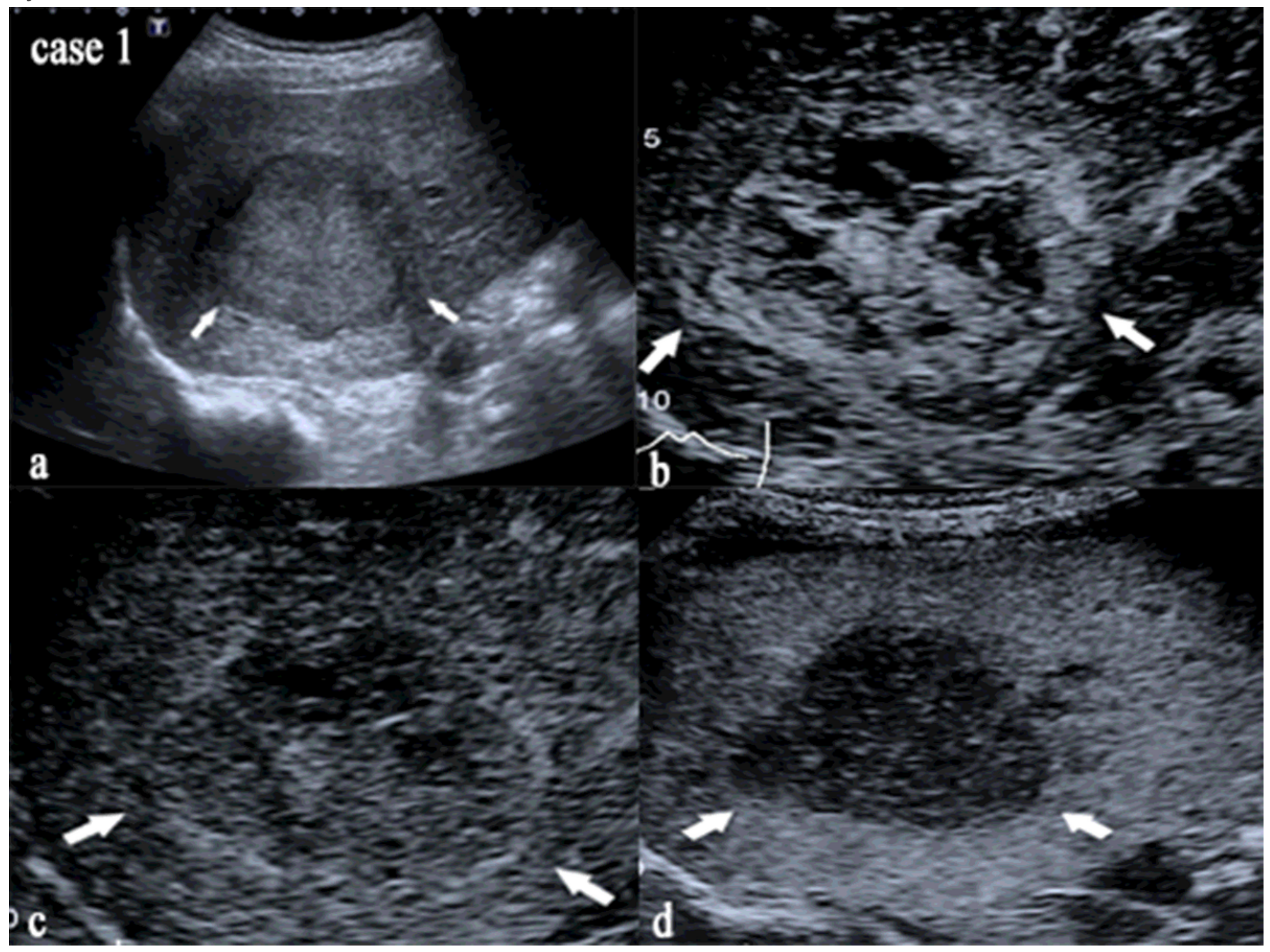

\section{Figure 2}

Case 1 was diagnosed as HCC histopathologically, assigned to LR-M by CE US LI-RADS with rim arterial phase hyperenhancement and portal venous phase early washout (time of washout was 52s), while diagnosed as HCC by U model (U-score $=0.927839594)$. a: image of nodule on US. b, c and d: images of nodule in arterial, portal venous and late phase on CE US. 


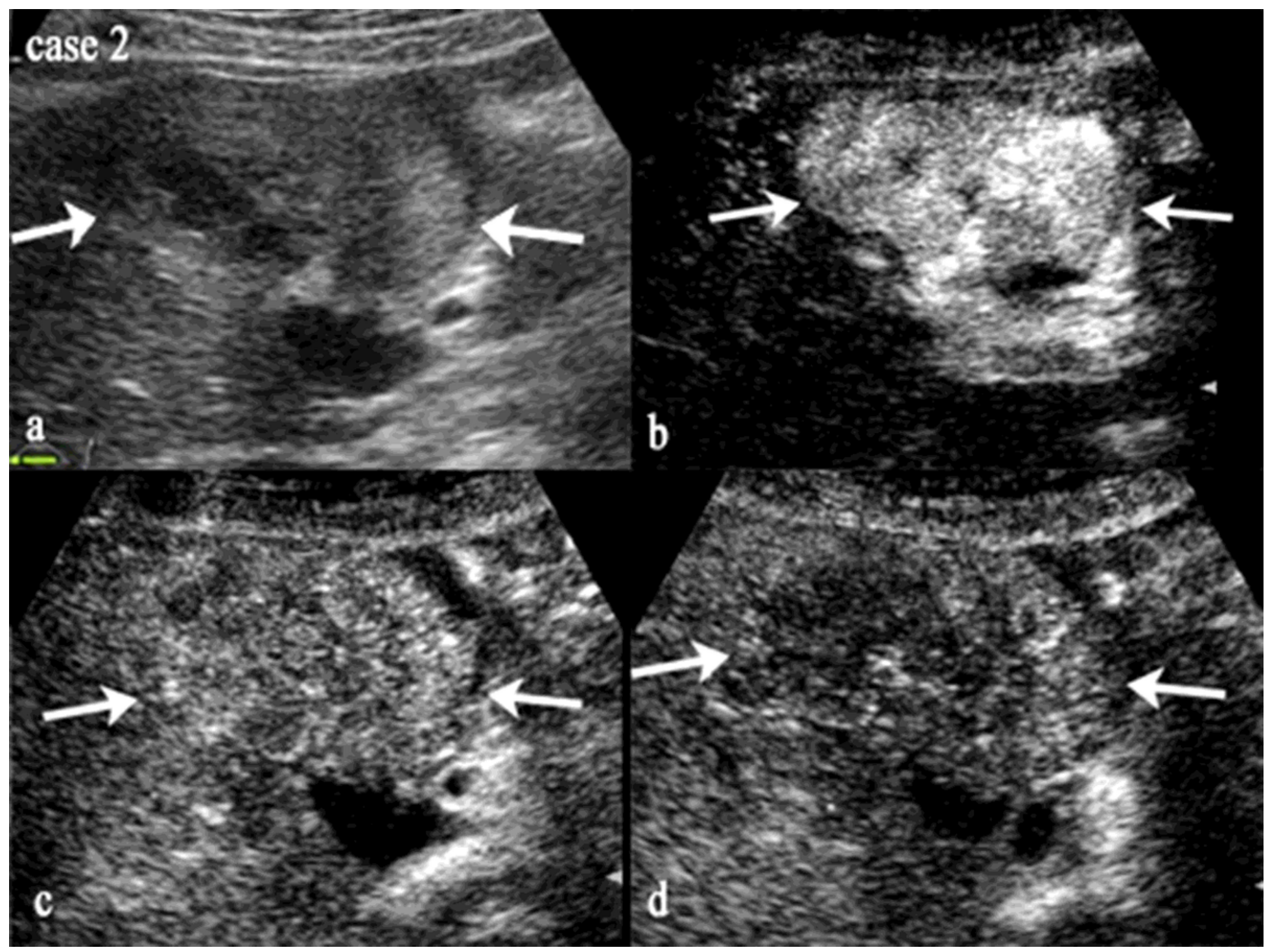

Figure 3

Case 2 was diagnosed as CHC histopathologically, assigned to LR- 5 by CE US LI-RADS with arterial phase hyperenhancement and portal venous phase mild, late washout (time of washout $>60 \mathrm{~s}$ ), while diagnosed as $\mathrm{CHC}$ by $\mathrm{U}$ model (U-score $=-0.236338237)$. a: image of nodule on US. $b, \mathrm{c}$ and $\mathrm{d}$ : images of nodule in arterial, portal venous and late phase on CE US. 